\title{
Inhibition of protein kinase $C$ enhances angiogenesis induced by platelet-derived growth factor $C$ in hyperglycemic endothelial cells
}

\author{
Junji Moriya and Napoleone Ferrara*
}

\begin{abstract}
Background: Diabetes is a risk factor for the development of cardiovascular diseases with impaired angiogenesis. We have previously shown that platelet-derived growth factor C (PDGF-C) and its receptor, PDGF receptor a (PDGFR-a) were downregulated in ischemic limbs of diabetic mice, although the underlying mechanisms remained elusive. Protein kinase C (PKC) is a family of serine/threonine kinases and is known to be involved in angiogenesis. The purpose of this study is to elucidate the mechanisms of how PDGF-C/PDGFR-a axis is impaired in diabetes.

Methods: Human umbilical vein endothelial cells (HUVECs) and human cardiac microvascular endothelial cells (HMVECS) cultured in normoglycemic or hyperglycemic conditions were examined. We also examined the effects of PKC inhibition on the PDGF-C/PDGFR-a axis in endothelial cells exposed to hyperglycemia.

Results: Hyperglycemia inhibited proliferation and decreased viability of both HUVECs and HMVECs. Hyperglycemic endothelial cells exhibited decreased PDGFR-a expression both at messenger RNA (mRNA) and protein levels, while there was no significant change in expression of PDGF-C. We also found that expression of PKC-a, one of the PKC isoforms, was increased in hyperglycemic endothelial cells and that inhibition of PKC upregulated PDGFR-a expression in these cells. Phosphorylation of extracellular signal-regulated kinase (ERK) and Akt induced by PDGF-C was significantly attenuated in hyperglycemic endothelial cells, whereas inhibition of PKC effectively reversed these inhibitory effects. Moreover, inhibition of PKC also promoted angiogenesis induced by PDGF-C in hyperglycemic endothelial cells, which was not observed in vascular endothelial growth factor-A (VEGF-A)-induced angiogenesis.

Conclusions: These findings suggest that downregulation of the PDGF-C/PDGFR- $a$ axis is involved in impaired angiogenesis of hyperglycemia through upregulation of PKC. Targeting PKC to restore PDGF-C signaling might be a novel therapeutic strategy for the treatment of vascular complications in diabetes.
\end{abstract}

Keywords: Platelet-derived growth factor C (PDGF-C), Diabetes, Therapeutic angiogenesis, Protein kinase C (PKC), Hyperglycemia, Endothelial cells

\section{Introduction}

There are increasing numbers of patients who suffer from ischemic cardiovascular diseases, and most of such patients have some risk factors. Among them, diabetes is known to have strong association with the development of cardiovascular diseases [1,2]. Indeed, vascular complications associated with diabetes may result from atherosclerosis of large vessels, causing cardiac, cerebral and peripheral vascular diseases [3,4]. Moreover, these

\footnotetext{
* Correspondence: nferrara@ucsd.edu

Moores Cancer Center, University of California, 3855 Health Sciences Dr, La Jolla, San Diego, CA 92093, USA
}

macrovascular atherosclerotic diseases are often refractory to conventional therapies, leading to increased morbidity and mortality among patients of diabetes [5-7]. Thus, it is important to establish novel therapeutic modalities for these patients.

Therapeutic angiogenesis is a relatively new and promising concept for treating patients with ischemic cardiovascular diseases [8-10]. It involves the use of angiogenic growth factors to promote development of collateral arteries. Vascular endothelial growth factor-A (VEGF-A) has 
been used as a major tool of this therapy since it is considered as the key regulator of angiogenesis [11-14]. However, several lines of evidence have shown that the benefits of this treatment are limited [15]. Indeed, the angiogenic response after ischemia is attenuated in patients with diabetes, interfering with the response to treatment $[16,17]$. Moreover, it has recently been reported that a ligandindependent VEGF receptor 2 (VEGFR2) signaling pathway is activated in diabetic endothelial cells, leading to impaired responses to exogenous VEGF-A and limited angiogenic events [18,19]. Collectively, these studies suggest that further basic research is needed to elucidate the mechanisms of angiogenesis, especially in diabetic state, to improve the overall outcome of therapeutic angiogenesis.

Platelet-derived growth factors (PDGFs) are potent mitogenic and migratory factors for many cell types of mesenchymal origin [20]. The PDGF family consists of four different polypeptide chains encoded by four different genes: PDGF-A, PDGF-B, and the more recently discovered PDGF-C and PDGF-D [21,22]. Among these, PDGF-C has been known to promote angiogenesis independently of VEGF-A [23]. Actually, inhibition of PDGF-C leads to reduced angiogenesis in experimental tumors refractory to anti-VEGF treatment [24] or to suppression of both choroidal and retinal neovascularization [25]. Moreover, PDGF-C has been reported to revascularize ischemic tissues by mobilizing endothelial progenitor cells or stimulating migration of endothelial cells [26]. We also have recently found that expression of PDGF-C and its receptor, PDGF receptor $\alpha$ (PDGFR- $\alpha$ ) was downregulated in ischemic limb tissues of diabetic mice, possibly contributing to impaired angiogenesis of diabetes. Moreover, introduction of PDGF-C significantly promoted revascularization in these mice [27]. Collectively, these studies suggest that PDGF-C may be an interesting candidate for therapeutic angiogenesis. However, the underlying mechanisms of how PDGF-C/PDGFR- $\alpha$ axis is impaired in diabetes still remain to be elucidated.

Therefore, we sought to investigate such mechanisms by using human endothelial cells (ECs) exposed to hyperglycemic condition. Hyperglycemia inhibited cell proliferation and decreased cell viability, which is in agreement with a previous study [28]. Moreover, we found that the expression of PDGFR- $\alpha$ was downregulated in hyperglycemic ECs both at mRNA and protein levels. We also found that upregulation of protein kinase C (PKC)- $\alpha$ expression was involved in decreased PDGFR- $\alpha$ expression in hyperglycemic ECs. Consistent with this finding, inhibition of PKC led to augmentation of intracellular signaling induced by PDGF-C, resulting in promotion of angiogenesis in hyperglycemic ECs. These findings suggest that downregulation of the PDGF-C/PDGFR- $\alpha$ axis is involved in impaired angiogenesis of diabetes through upregulation of PKC. Targeting PDGF-C and PKC might be a novel strategy for therapeutic angiogenesis in the diabetic state.

\section{Materials and methods Reagents}

Recombinant human VEGF-A and PDGF-C were from Peprotech (Rocky Hill, NJ) and Sigma-Aldrich (St. Louis, $\mathrm{MO}$ ), respectively. D-mannitol and d-glucose were from Sigma-Aldrich. Trypan blue solution was from GE Healthcare Life Sciences (Little Chalfont, UK). Bisindolylmaleimide I (Bis I) was purchased from Cell Signaling Technology (Danvers, MA). Sulforaphane was from Trevigen (Gaithersburg, MD).

\section{Cells}

Human umbilical vein endothelial cells (HUVECs), human cardiac microvascular endothelial cells (HMVECs), and human aortic smooth muscle cells (AoSMCs) were purchased from Lonza (Walkersville, MD). HUVECs were grown in EGM-2 medium (Lonza), HMVECs in EGM-2-MV medium (Lonza), and AoSMCs in SmGM-2 medium (Lonza) on gelatin-coated tissue culture dishes (BD Bioscience, Becton, NJ), respectively. Cells from fewer than 5 generations were used for all experiments. High-glucose cultures were grown in the presence of 30 $\mathrm{mM}$ glucose for a minimum of 5 days and then subjected to further analysis. The total cell numbers were calculated by a Countess automated cell counter (Life Technologies, Carlsbad, CA). For signaling pathway analysis and tube formation analysis, HUVECs were serum-starved for 15 hours in EBM-2 medium (Lonza) supplemented with 0.1\% fetal bovine serum (Omega Scientific, Tarzana, CA) before being treated with test substances.

\section{RNA analysis}

Total RNA was extracted by an RNeasy Plus Mini kit (Qiagen, Valencia, CA) according to the manufacturer's instructions. cDNA was prepared using High Capacity cDNA Reverese Transcription Kits (Life Technologies). Quantitative real-time polymerase chain reaction (PCR) was performed by the ViiA7 Real-Time PCR system (Life Technologies) with the Taqman Gene Expression Assays and the Taqman Fast Advanced Master Mix (Life Technologies). Glyceraldehyde-3-phosphate dehydrogenase (GAPDH) messenger RNA was used as an endogenous control for quantitative real-time PCR analyses. At least three biological replicates were included in each analysis.

\section{Western blot analysis}

Whole cell lysates were prepared in RIPA buffer (Thermo Scientific, Rockford, IL) with Halt Protease and Phosphatase Inhibitor Cocktail (Thermo Scientific). The lysates $(20$ to $40 \mu \mathrm{g})$ were resolved by SDS-PAGE. The 
proteins were then transferred into a polyvinylidene difluoride membrane (Bio-Rad, Hercules, CA) and incubated with the primary antibody followed by anti-rabbit IgG-horseradish peroxidase antibody or anti-mouse IgGhorseradish peroxidase antibody (GE Healthcare, Little Chalfont, UK). Specific proteins were detected by the enhanced chemiluminescent substrate (Thermo Scientific). The primary antibodies used for Western blotting were as follows: anti-PDGFR $\alpha$ antibody (951, sc-431), anti-PKC $\alpha$ antibody (C-20, sc-208), anti-PKC $\beta$ II antibody (C-18, sc-210), anti-PKC $\delta$ antibody (C-17, sc-213) (Santa Cruz Biotechnology, Santa Cruz, CA), anti-phospho-extracellular signal-regulated kinase (ERK) antibody (20G11, \#4376), anti-ERK antibody (137F5, \#4695), anti-phospho-Akt antibody (D9E, \#4060), anti-Akt antibody (C67E7, \#4691) (Cell Signaling Technology), and anti- $\beta$-actin antibody (AC-74, A2228) (Sigma-Aldrich).

\section{Tube formation assay}

The tube formation assay was performed using a commercially available kit (In Vitro Angiogenesis Tube Formation Assay Kit, Trevigen, \#3470-096-K) according to the manufacturer's instructions. Briefly, growth factor-reduced basement membrane extract solution in a 96-well plate was allowed to form a reconstituted matrix for one hour at $37^{\circ} \mathrm{C}$. HUVECs were seeded at $1.5 \times 10^{4}$ per well and cultured for up to 24 hours in the presence or absence of different kinds of test substances. Capillary-like tube formation was assessed by photography under a stereoscopic microscope (Zeiss, Oberkochen, Germany) at a $\times 80 \mathrm{mag}$ nification. Total tube length was analyzed by using Image J software (NIH, Bethesda, MD).

\section{Statistical analysis}

The data are shown as means \pm standard error of the mean. Differences between two groups were analyzed by a two-sided Student $t$-test. One-way analysis of variance (ANOVA) was used for multiple group comparison followed by the Bonferroni procedure for comparison of means. All experiments were repeated at least three times. In all analyses, $P$ values of $<0.05$ were considered statistically significant.

\section{Results}

Hyperglycemia inhibits cell proliferation and decreases cell viability of endothelial cells

We first examined the effect of hyperglycemia on proliferation and viability of endothelial cells (ECs). Since we confirmed that the total number of cells and the ratio of cells positive for trypan blue staining exposed to $24.5 \mathrm{mM}$ $\mathrm{d}$-mannitol in normoglycemic (5.5 $\mathrm{mM} \mathrm{d}$-glucose) conditions were not different from those in control cultures (Additional file 1: Figure S1), we used $24.5 \mathrm{mM} \mathrm{d}$-mannitol as an osmotic control for in all further experiments. We examined two types of human EC; human umbilical vein endothelial cells (HUVECs) and human cardiac microvascular endothelial cells (HMVECs). We plated HUVECs and HMVECs in normoglycemic or hyperglycemic $(30 \mathrm{mM}$ d-glucose) conditions and cultured them for 5 days. As shown in Figure 1A, HUVECs or HMVECs cultured for 5 days in such hyperglycemic condition showed reduced increases in total cell numbers, compared to normoglycemic conditions. Moreover, the ratio of cells positive for trypan blue staining, which are thought to be dead cells, was significantly increased in HUVECs and HMVECs cultured in hyperglycemic condition (Figure 1B). These results suggest that hyperglycemia inhibits cell proliferation and decreases cell viability of ECs.

\section{Expression of PDGFR- $a$ is downregulated in hyperglycemic endothelial cells}

We have previously reported that expression of PDGF-C or PDGFR- $\alpha$ after ischemia is decreased in diabetic mice, leading to impaired angiogenesis [27]. Thus, we sought to examine messenger RNA (mRNA) levels of these angiogenic factors in hyperglycemic ECs (HUVECs and HMVECs) by quantitative real-time PCR (qRT-PCR) analysis. We found that compared to normoglycemic conditions, expression of PDGFR- $\alpha$ was markedly decreased in hyperglycemic ECs (Figure 2A). VEGFR2 expression was also modestly decreased in hyperglycemic ECs, although we did not observe any differences in PDGF-C or VEGFA expression compared to normoglycemia (Figure 2A). After confirming that PDGFR- $\alpha$ expression was almost comparable in normoglycemic ECs treated with or without $24.5 \mathrm{mM}$ d-mannitol (Additional file 2: Figure S2A, B), we performed Western blot analysis to examine the protein expression of PDGFR- $\alpha$ in hyperglycemic ECs. Consistent with our qRT-PCR results, the expression of PDGFR- $\alpha$ in hyperglycemic ECs was markedly decreased compared to normoglycemic, mannitol-treated ECs (Figure 2B, C). These results indicate that the expression of PDGFR- $\alpha$ is downregulated in hyperglycemic ECs both in mRNA and protein levels.

\section{Upregulation of PKC leads to decreased PDGFR-a expression in hyperglycemic endothelial cells}

Hyperglycemia has been reported to activate members of the protein kinase C (PKC) family, some of which are implicated in angiogenesis [29]. We hypothesized that PKC might be implicated in the decreased expression of PDGFR- $\alpha$ in hyperglycemic ECs. To test this hypothesis, we first examined the expression of PKC- $\alpha$, PKC- $\beta \mathrm{II}$, and $\mathrm{PKC}-\delta$, which are known to be expressed in ECs [30]. Because HUVECs and HMVECs showed the same tendency toward the total number of cells, ratio of cells positive for trypan blue staining, and expression of PDGFR- $\alpha$ exposed to hyperglycemic conditions (Figures 1, 2), 


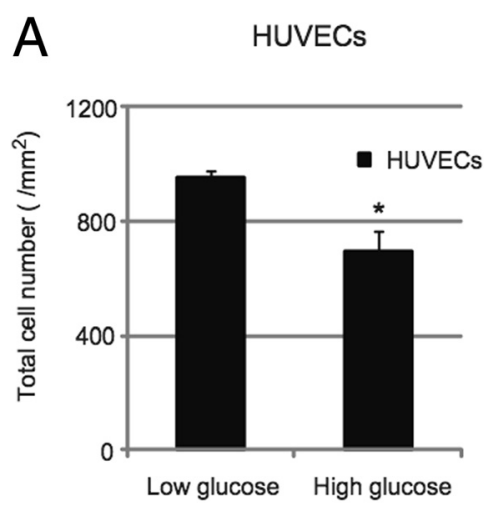

$\mathrm{B}$

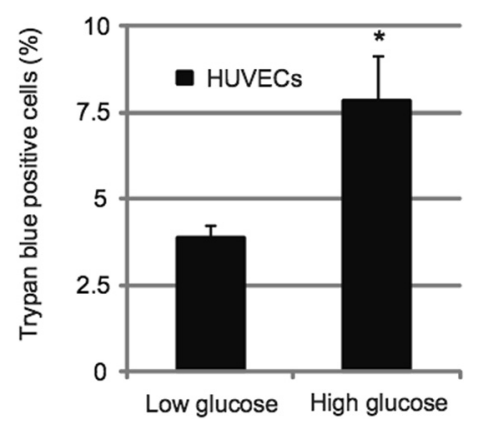

HMVECs

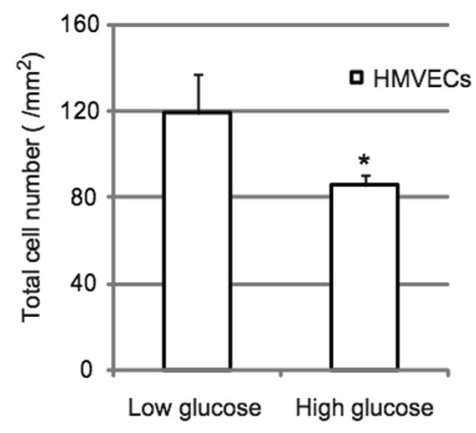

HMVECs

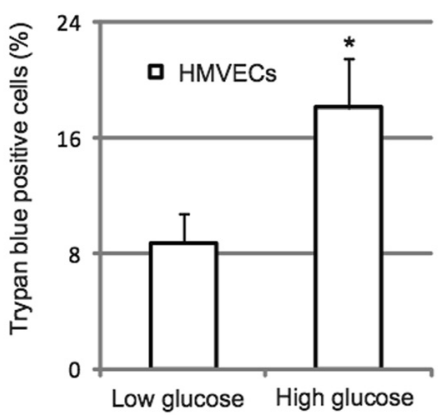

Figure 1 Effects of hyperglycemia on endothelial cells. A: HUVECs or HMVECs were treated with 5.5 mM (Low) or 30 mM (High) glucose and total cell numbers were calculated. ${ }^{*} P<0.05$ vs Low glucose $(n=4$ for each group). Data represent means \pm standard error of the mean. B: Ratio of trypan blue positive HUVECs or HMVECs treated with $5.5 \mathrm{mM}$ (Low) or $30 \mathrm{mM}$ (High) glucose. ${ }^{*} P<0.05$ vs Low glucose ( $\mathrm{n}=4$ for each group). Data represent means \pm standard error of the mean.

and considering that HUVECs have been widely used to elucidate molecular mechanisms of diabetic state in ECs $[31,32]$, we used only HUVECs for further experiments. We found that hyperglycemia significantly upregulated the protein expression of PKC- $\alpha$ in HUVECs (Figure 3A, B). On the other hand, the expression of PKC- $\beta \mathrm{II}$ or PKC- $\delta$ was not significantly different between normoglycemic and hyperglycemic ECs (Figure 3B), although we could not exclude the possibility that these isoforms were still activated in hyperglycemia. Next, we investigated the effects of inhibition of PKC. We treated normoglycemic and hyperglycemic HUVECs with bisindolylmaleimide I (Bis I), an inhibitor of PKC [33], and examined the expression of PKC- $\alpha$ and PDGFR- $\alpha$ by Western blot analysis. Consistent with previous obsevations [34], we confirmed that treatment with Bis I significantly downregulated the expression of $\mathrm{PKC}-\alpha$ both in normoglycemic and hyperglycemic HUVECs (Figure 3C, D). Interestingly, downregulation of PDGFR- $\alpha$ expression was completely abolished by treatment with Bis I in hyperglycemic ECs (Figure 3C, D). We also confirmed that the effect of Bis I had some dosedependent tendency to the reduced expression of PKC- $\alpha$, although it was not statistically significant (Additional file 3: Figure S3A). Moreover, we found that longer exposure to Bis I significantly upregulated the expression of PDGFR- $\alpha$ even in normoglycemic ECs (Additional file 3: Figure S3B). These results suggest that upregulation of $\mathrm{PKC}$ expression induced by hyperglycemia is involved in the downregulation of PDGFR- $\alpha$ expression in HUVECs.

Inhibition of PKC leads to increased intracellular signaling induced by PDGF-C in endothelial cells

Next we investigated whether downregulation of PDGFR- $\alpha$ in hyperglycemic ECs actually affects downstream signaling induced by PDGF-C. We found that PDGF-Cinduced phosphorylation of Akt and ERK, both of which are crucial kinases in the intracellular signaling pathway for this growth factor, was abolished in hyperglycemic HUVECs, which is in contrast to normoglycemic HUVECs (Figure 4A, B). However, pretreatment with Bis I significantly increased phosphorylation of Akt in hyperglycemic HUVECs and phosphorylation of ERK in hyperglycemic and normoglycemic HUVECs following PDGF-C stimulation (Figure 4A, B). We also observed decreased 

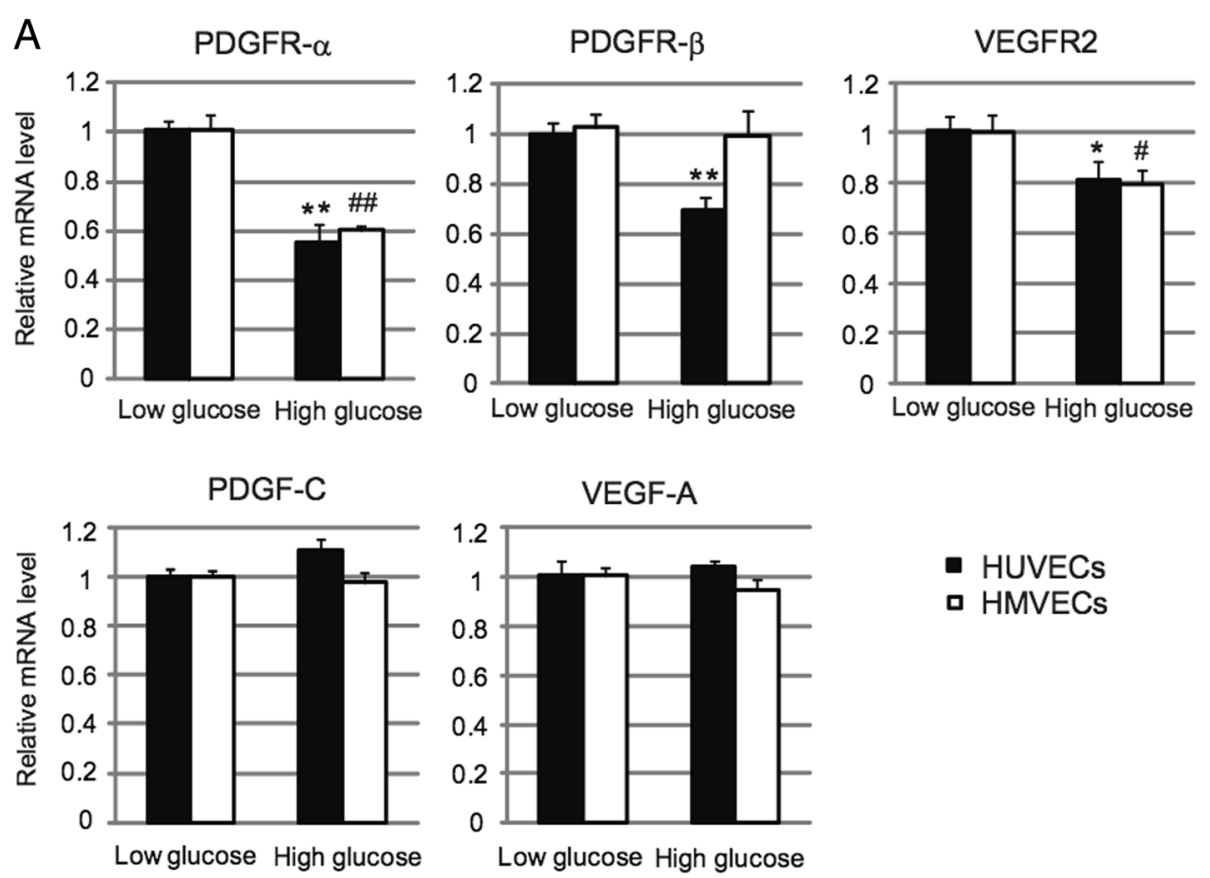

- HUVECs

口 HMVECs

B

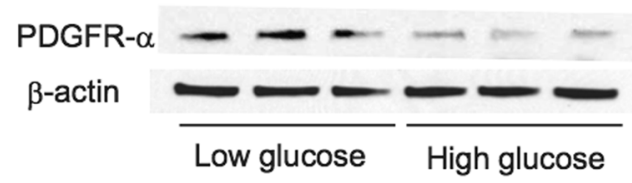

C
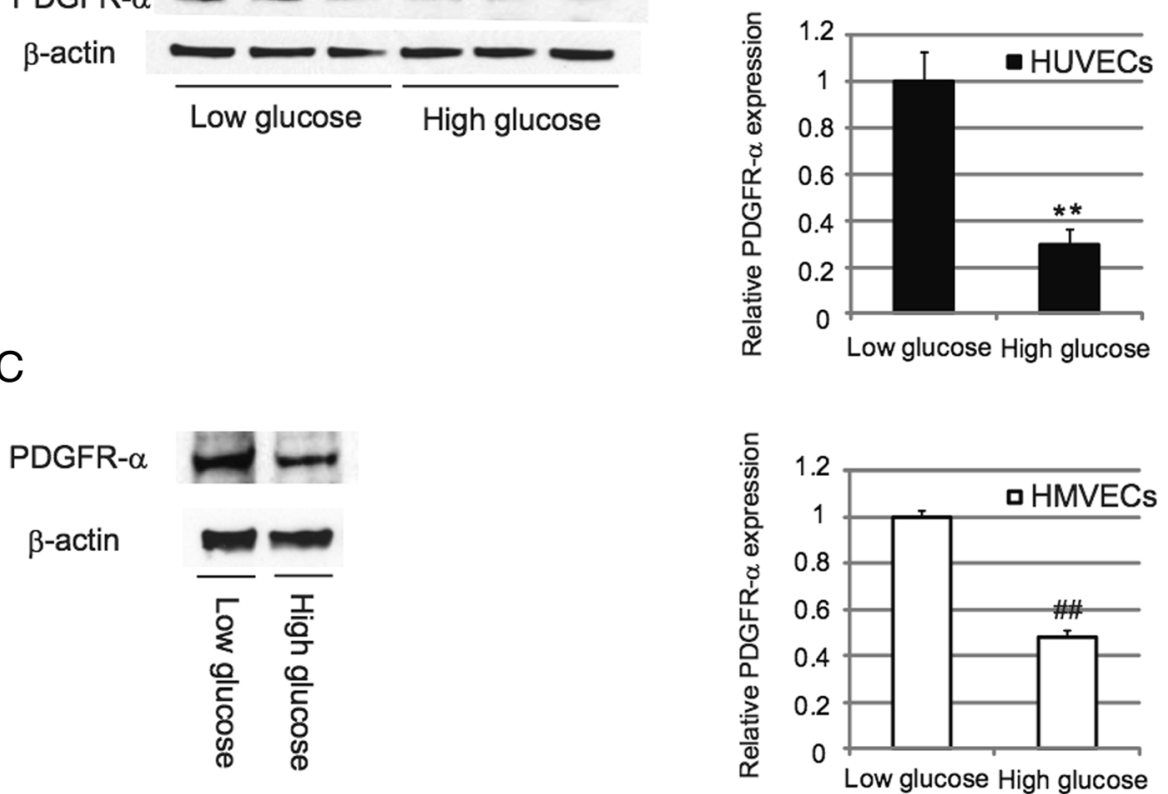

Figure 2 Expression of PDGFR- $\alpha$ is downregulated in hyperglycemic endothelial cells. A: PDGFR- $\alpha$, PDGFR- $\beta$, VEGFR2, PDGF-C, and VEGF- $A$ mRNA levels in HUVECs or HMVECs treated with $5.5 \mathrm{mM}$ (Low) or $30 \mathrm{mM}$ (High) glucose were assessed by quantitative real-time PCR. ${ }^{*} P<0.05$, ${ }^{*} P<0.01$ vs Low glucose HUVECs ( $n=6$ for each group). $\# P<0.05$, \#\#P<0.01 vs Low glucose HMVECs $(n=6$ for each group). Data represent means \pm standard error of the mean. B: Western blot analysis of PDGFR-a expression in HUVECs treated with 5.5 mM (Low) or 30 mM (High) glucose. Relative quantification data are also shown (right). ${ }^{* *} P<0.01$ vs Low glucose ( $n=6$ for each group). Data represent means \pm standard error of the mean. C: Western blot analysis of PDGFR-a expression in HMVECs treated with $5.5 \mathrm{mM}$ (Low) or $30 \mathrm{mM}$ (High) glucose. Relative quantification data are also shown (right). \#\#P<0.01 vs Low glucose ( $n=8$ for each group). Data represent means \pm standard error of the mean.

VEGF-A-induced phosphorylation of Akt and ERK in hyperglycemic HUVECs (Additional file 4: Figure S4). However, unlike PDGF-C, treatment with Bis I in hyperglycemic HUVECs did not increase phosphorylation of Akt or ERK induced by VEGF-A (Additional file 4: Figure S4). Moreover, tretament with Bis I significantly 


\section{A}

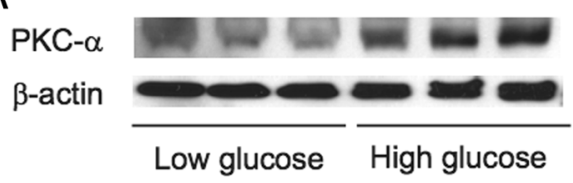

B

PKC- $\alpha$

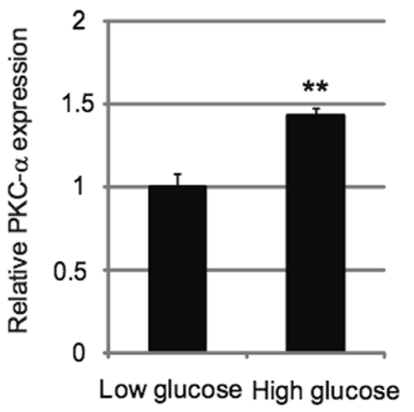

PKC- $\beta$ II

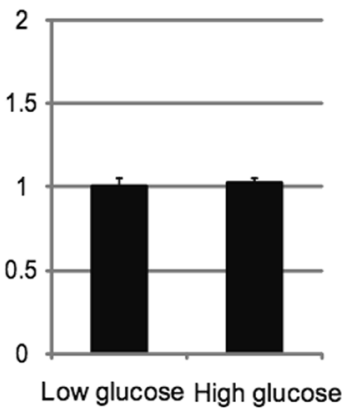

PKC- $\delta$

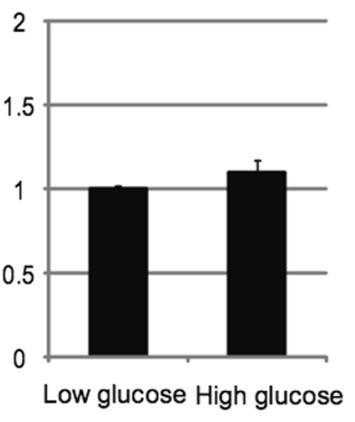

C

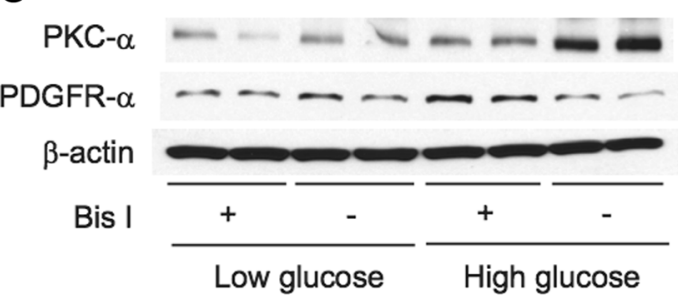

D
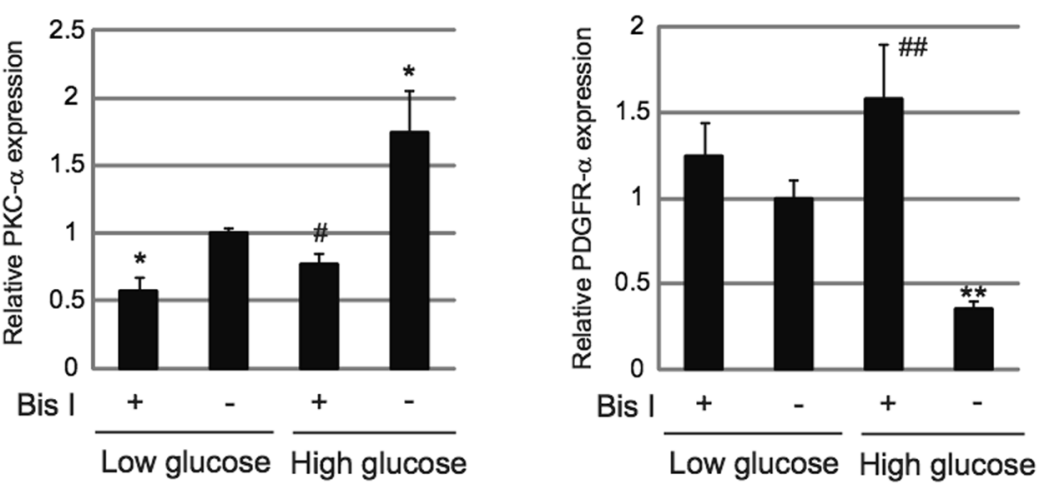

Figure 3 Upregulation of PKC leads to decreased PDGFR-a expression in hyperglycemic endothelial cells. A: Western blot analysis of PKC-a in HUVECs treated with $5.5 \mathrm{mM}$ (Low) or $30 \mathrm{mM}$ (High) glucose. B: Relative quantification data of Western blot analysis of PKC-a, PKC- $\beta 11$, and PKC- $\delta$ in HUVECs treated with $5.5 \mathrm{mM}$ (Low) or $30 \mathrm{mM}$ (High) glucose. ${ }^{* *} P<0.01$ vs Low glucose ( $\mathrm{n}=4$ for each group). Data represent means \pm standard error of the mean. C: HUVECs exposed to $5.5 \mathrm{mM}$ (Low) or $30 \mathrm{mM}$ (High) glucose were treated with or without bisindolylmaleimide I (Bis I) at a concentration of $4 \mu \mathrm{M}$ for 30 minutes and then were subject to Western blot analysis for PKC-a and PDGFR-a expression. D: Relative expression levels of PKC- $a$ and PDGFR-a in the experiment of Figure 3C. ${ }^{*} P<0.05,{ }^{* *} P<0.01$ vs Low glucose, Bis I (-) group. \#P<0.05, \#\#P<0.01 vs High glucose, Bis I (-) group. $n=4$ for each group. Data represent means \pm standard error of the mean.

reduced VEGF-A-induced phosphorylation of Akt and ERK in normoglycemic HUVECs (Additional file 4: Figure S4). Collectively, these results suggest that inhibition of PKC could potentiate the intracellular signaling induced by PDGF-C, but not VEGF-A, in hyperglycemic HUVECs.
Inhibition of PKC promotes angiogenesis induced by PDGF-C, but not VEGF-A, in hyperglycemic endothelial cells To investigate the effects of PKC inhibition on angiogenesis, we performed tube formation assays using HUVECs in normoglycemic and hyperglycemic conditions. Sulforaphane was used as a negative control for the assays. 
A

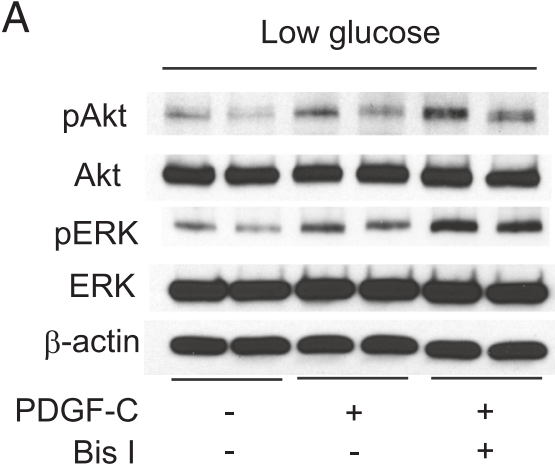

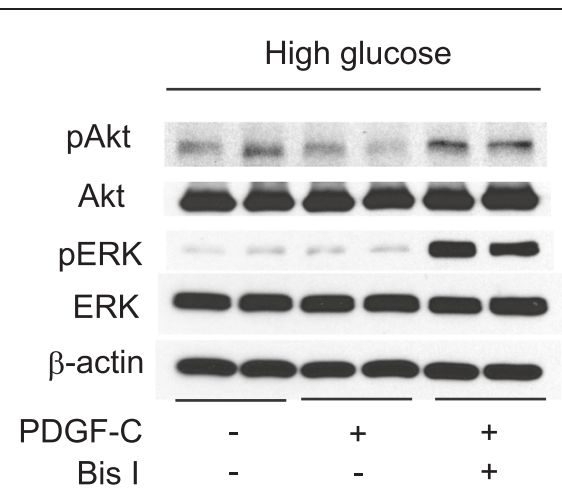

B

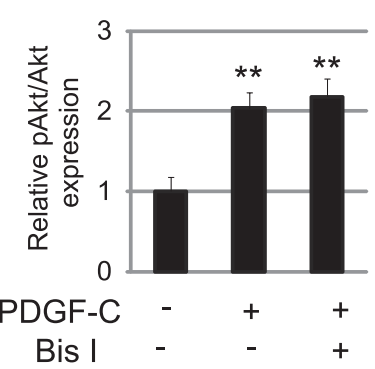

High glucose

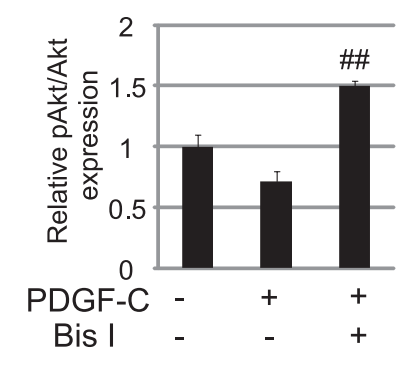

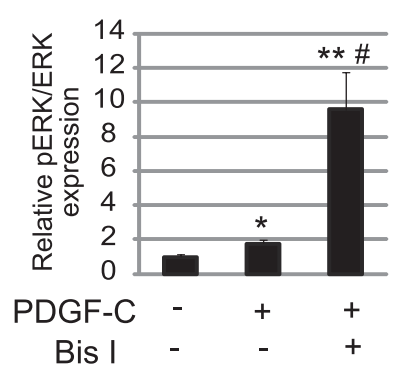

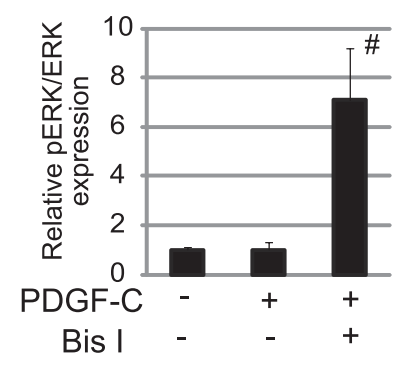

Figure 4 Inhibition of PKC potentiates intracellular signaling induced by PDGF-C in endothelial cells. A: HUVECs exposed to 5.5 mM (LoW) or $30 \mathrm{mM}$ (High) glucose were treated with PDGF-C (50 ng/mL) or PDGF-C + Bis I $(4 \mu \mathrm{M})$ and analyzed for signaling molecules by Western blot analysis. B: Relative expression levels of pERK and pAkt in the experiment of Figure 4A. Results are shown as the ratio of pAkt to Akt densities, and pERK to ERK densities, respectively. ${ }^{*} P<0.05$, ${ }^{* *} P<0.01$ vs PDGF-C (-), Bis I (-) group. \#P<0.05, \#\#P<0.01 vs PDGF-C (+), Bis I (-) group. $\mathrm{n}=4$ for each group. Data represent means \pm standard error of the mean.

We found that both PDGF-C and VEGF-A treatment markedly increased tube formation in normoglycemic HUVECs, while this increase was blunted but still significant in hyperglycemic HUVECs (Figure 5A-C). Intriguingly, treatment with Bis I significantly augmented PDGF-C-induced tube formation in hyperglycemic HUVECs (Figure 5B, C). However, treatment with Bis I did not increase or even significantly decreased VEGF-Ainduced tube formation (Figure 5A-C). Treatment with Bis I alone did not affect tube formation, both in normoglycemic and hypeglycemic HUVECs (Figure 5A-C). These results suggest that $\mathrm{PKC}$ inhibition promotes angiogenesis induced by PDGF-C, but not VEGF-A, in hyperglycemic HUVECs possibly by upregulating PDGF-C/ PDGFR- $\alpha$ axis.

\section{Discussion}

PDGFs have a variety of effects in many cell types. Among the four members of the PDGF family, PDGF-B and PDGF-C have been extensively characterized for their role in vessel maturation and growth. While PDGF-B requires other angiogenic factors like VEGF-A to induce neovascularization, PDGF-C has the potential to promote revascularization after ischemia, independently of other factors $[23,26]$. Moreover, we recently reported that expression of PDGF-C or PDGFR- $\alpha$ was downregulated in ischemic 


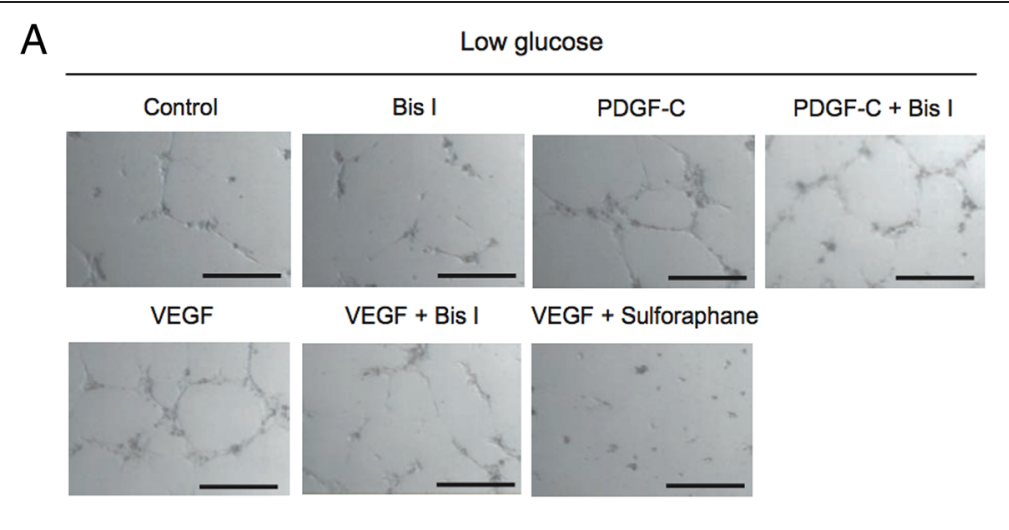

B

High glucose
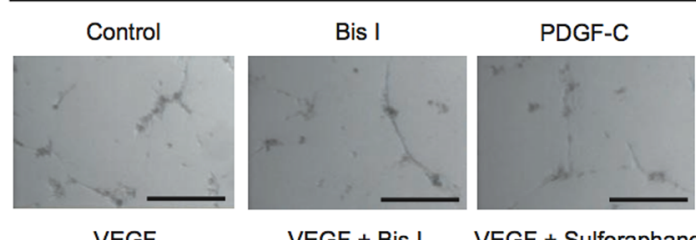

PDGF-C + Bis I

VEGF

VEGF + Bis I

VEGF + Sulforaphane
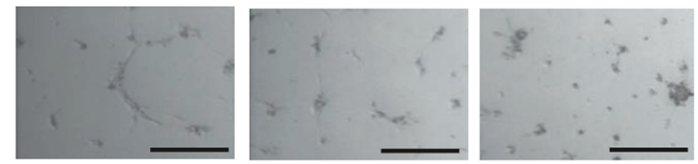

C

Low glucose
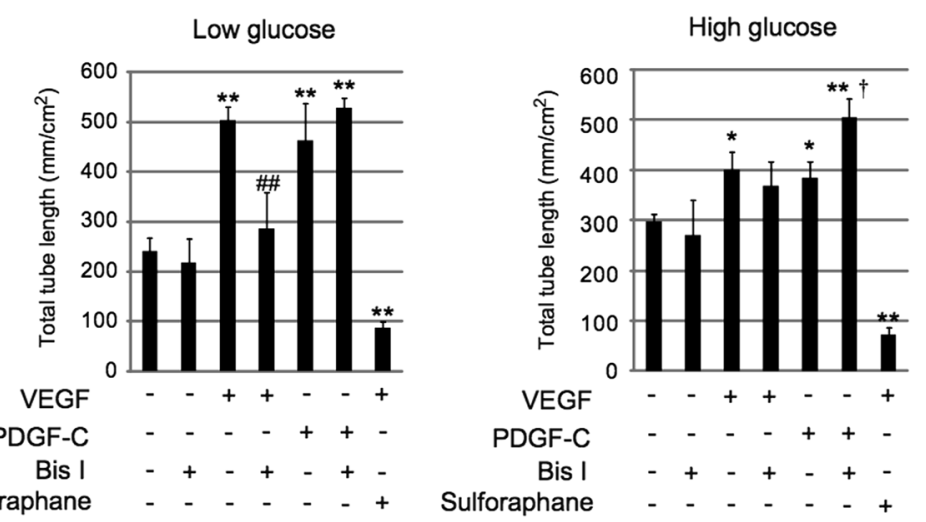

Figure 5 Inhibition of PKC promotes angiogenesis induced by PDGF-C, but not VEGF-A, in hyperglycemic endothelial cells.

A, B: Photographs show tube formation in HUVECs exposed to $5.5 \mathrm{mM}$ (Low) (A) or $30 \mathrm{mM}$ (High) (B) glucose in the presence of Bis I (4 $\mu \mathrm{M}$ ), PDGF-C (50 ng/mL), PDGF-C + Bis I, VEGF (50 ng/mL), VEGF + Bis l, and VEGF + Sulforaphane (5 $\mu \mathrm{M})$. PBS treatment served as control (Control). Sulforaphane was used as a negative control for the assay. Scale bar $=300 \mu \mathrm{m}$. C: Total tube length was analyzed in the experiment of Figure $5 \mathrm{~A}$ (left) and $5 B$ (right). ${ }^{*} P<0.05,{ }^{*} P<0.01$ vs Control $\operatorname{VEGF}(-)$, PDGF-C(-), Bis I(-), and Sulforaphane(-)) group. \#\#P<0.01 vs VEGF(+), PDGF-C(-), Bis I (-), and Sulforaphane (-) group. $+P<0.05$ Vs VEGF(-), PDGF-C(+), Bis I (-), and Sulforaphane (-) group. $n=5 \sim 9$ for each group. Data represent means \pm standard error of the mean.

tissues of diabetic mice, resulting in the impaired blood flow recovery after ischemia [27]. Also, a recent study reported that PDGF signaling is critically involved in the pathogenesis of diabetes [35]. On the other hand, a clinical study revealed that there were no significant differences in the serum concentrations of VEGF between control subjects and diabetic patients [36]. Taken together, these findings support the hypothesis that PDGF-C might be a promising new target for the treatment of vascular complications related to diabetes.

The present study sought to investigate how the PDGF-C/PDGFR- $\alpha$ axis is downregulated using cultured human ECs exposed to hyperglycemia. We found that hyperglycemia led to inhibition of cell proliferation, decreased cell viability, and reduced angiogenic responses to VEGF-A in ECs (Figures 1A-B, 5A-C). These results 
are consistent with our previous in vivo studies in diabetic mice showing reduced blood flow recovery and neovascularization after ischemia even with introduction of VEGF-A [27,31].

We also show here that expression of PDGFR- $\alpha$ was significantly downregulated in hyperglycemic ECs, while expression of PDGF-C was almost unchanged between normoglycemic and hyperglycemic ECs (Figure 2A). On the other hand, we found that expression of PDGF-C was significantly downregulated both at baseline and after ischemia in limb tissues of diabetic mice in our previous in vivo study [27]. Since in vivo murine limb tissues contain various cell types besides ECs such as vascular smooth muscle cells, fibroblasts, and skeletal muscle cells, it is possible that decreased PDGF-C expression occurs in cell types other than ECs in diabetic limbs. Indeed, we observed that the expression of PDGF-C was decreased in hyperglycemic human aortic smooth muscle cells (AoSMCs) in vitro (data not shown), suggesting that SMCs might be more responsible for the decreased expression of PDGF-C in vivo as well. Further studies are required to identify the cellular localization of the decreased PDGF-C and/or PDGFR- $\alpha$ expression in vivo.

Our results also suggest that $\mathrm{PKC}$ is critically involved in the decreased PDGFR- $\alpha$ expression in hyperglycemic ECs. We found that expression of PKC- $\alpha$ was significantly upregulated in hyperglycemic ECs (Figure 3A, B). $\mathrm{PKC}$ is a member of a large family of serine/threonine kinases and is known to be involved in angiogenesis [37]. Moreover, PKC is also implicated in the increased risk of atherosclerosis in diabetes [38]. However, the role of $\mathrm{PKC}$ for angiogenesis still remains elusive because the effect of PKC on angiogenesis seems to be different or even opposite depending on PKC isoforms [39]. Moreover, there are some conflicting reports indicating that PKC- $\alpha$ promotes $[40,41]$ or inhibits [42] angiogenic activity of ECs, and it has been known that inhibition of PKC leads to suppression of VEGF release from platelets [43]. We show here that inhibition of PKC by Bis I leads to increased PDGFR- $\alpha$ expression, resulting in potentiation of intracellular signaling, and augmentation of angiogenesis induced by PDGF-C, but not by VEGF-A (Figures 3, 4, 5). Based on these findings, we speculate that the complexity and apparent discrepancies in the effects of PKC on angiogenesis stem not only from different PKC isoforms but also from downstream molecular targets of PKC in ECs. Further studies are needed to confirm whether $\mathrm{PKC}$ differentially regulates angiogenesis induced by PDGF-C and VEGF-A signaling, in addition to the effects of specific inhibition or activation of PKC- $\alpha$ on angiogenesis induced by PDGF-C in the diabetic state.

In summary, the present study shows that downregulation of PDGF-C/PDGFR- $\alpha$ axis is involved in impaired endothelial cell functions in hyperglycemia at least in part through upregulation of PKC. Therefore, targeting $\mathrm{PKC}$ to restore PDGF-C signaling might be anovel therapeutic option for the treatment of vascular complications in diabetic patients.

\section{Additional files}

\begin{abstract}
Additional file 1: Figure S1. Effects of mannitol on HUVECS. HUVECS were treated with $\mathrm{d}$-mannitol at the concentration of $24.5 \mathrm{mM}$ in normoglycemic (5.5 mM glucose) conditions for 5 days (Mannitol). Total cell number of (left) and the ratio of cells positive for trypan blue (right) were analyzed. HUVECs cultured in normoglycemic conditions served as control (Control). $n=5$ for each group. Data represent means \pm standard error of the mean
\end{abstract}

Additional file 2: Figure S2. Treatment with mannitol does not affect expression of PDGFR-a in endothelial cells. A: HUVECs were treated with or without $24.5 \mathrm{mM}$ d-mannitol in normoglycemic conditions and then subjected to Western blot analysis for PDGFR-a. Relative quantification data are also shown (right). $n=3$ for each group. Data represent means \pm standard error of the mean. B: HMVECs were treated with or without $24.5 \mathrm{mM}$ d-mannitol in normoglycemic condition and then subjected to Western blot analysis for PDGFR-a. Relative quantification data are also shown (right). $n=3$ for each group. Data represent means \pm standard error of the mean.

Additional file 3: Figure S3. Effect of PKC inhibition on expression of PKC- $a$ and PDGFR- $a$ in endothelial cells. A: HUVECs exposed to $5.5 \mathrm{mM}$ (Low) or $30 \mathrm{mM}$ (High) glucose were treated or not bisindolylmaleimide I (Bis I) at the concentration of 2 or $4 \mu \mathrm{M}$ for 30 minutes and then were subject to Western blot analysis for PKC-a and PDGFR-a expression. Relative expression levels of PKC- $a$ and PDGFR- $a$ are shown. ${ }^{*} P<0.05$ vs Low glucose, Bis I (-) group. \#\#P<0.01 vs High glucose, Bis I (-) group. $n=4$ for each group. Data represent means \pm standard error of the mean. B: HUVECs exposed to $5.5 \mathrm{mM}$ (Low) or $30 \mathrm{mM}$ (High) glucose were treated with or without Bis I at a concentration of $4 \mu \mathrm{M}$ for 120 minutes and then were subject to Western blot analysis for PKC- $a$ and PDGFR- $a$ expression. Relative expression levels of PKC- $a$ and PDGFR- $a$ are shown. ${ }^{*} P<0.05,{ }^{*} P<0.01$ vs Low glucose, Bis I (-) group. \#P<0.05, \#\#P<0.01 vs High glucose, Bis I (-) group. $n=4$ for each group. Data represent means \pm standard error of the mean.

Additional file 4: Figure S4. Effects of PKC inhibition on intracellular signaling induced by VEGF-A in normoglycemic or hyperglycemic endothelial cells. HUVECs exposed to $5.5 \mathrm{mM}$ (Low) or $30 \mathrm{mM}$ (High) glucose were treated with VEGF-A alone $(50 \mathrm{ng} / \mathrm{mL})$ or VEGF-A + Bis I $(4 \mu \mathrm{M})$ and analyzed for the VEGF-A signaling pathways by Western blot analysis. Relative expression levels of pERK and pAkt are shown as the ratio of pAkt to Akt densities, and pERK to ERK densities, respectively. ${ }^{*} P<0.05$, ${ }^{*} P<0.01$ vs VEGF-A (-), Bis I (-) group. \#P<0.05 VS VEGF-A (+), Bis I (-) group. $n=5 \sim 6$ for each group. Data represent means \pm standard error of the mean.

\section{Competing interests}

The authors declare that they have no competing interests.

\section{Authors' contributions}

JM and NF designed and performed experiments. JM analyzed the data, performed the statistical analysis and wrote the manuscript. NF reviewed and edited the manuscript. Both authors read and approved the final manuscript.

\section{Acknowledgement}

We thank H. Xin, C. Zhong, W. Liang and N. Protopsaltis for helpful discussions and advice. This work was supported by start-up funds of University of California, San Diego (to N.F.) and by grants from the Uehara Memorial Foundation, Kanae Foundation for the Promotion of Science, and the NOVARTIS Foundation for Gerontological Research (to J.M.). 
Received: 28 August 2014 Accepted: 20 January 2015 Published online: 07 February 2015

\section{References}

1. Eckel RH, Kahn R, Robertson RM, Rizza RA. Preventing cardiovascular disease and diabetes: a call to action from the American Diabetes Association and the American Heart Association. Circulation. 2006;113(25):2943-6.

2. Van Gaal LF, Mertens IL, De Block CE. Mechanisms linking obesity with cardiovascular disease. Nature. 2006;444(7121):875-80.

3. Nathan DM. Long-term complications of diabetes mellitus. N Engl J Med 1993:328(23):1676-85.

4. Fox CS, Coady S, Sorlie PD, Levy D, Meigs JB, D'Agostino Sr RB, et al. Trends in cardiovascular complications of diabetes. JAMA. 2004;292(20):2495-9.

5. Haffner SM, Lehto S, Ronnemaa T, Pyorala K, Laakso M. Mortality from coronary heart disease in subjects with type 2 diabetes and in nondiabetic subjects with and without prior myocardial infarction. N Engl J Med. 1998;339(4):229-34.

6. Cho E, Rimm EB, Stampfer MJ, Willett WC, Hu FB. The impact of diabetes mellitus and prior myocardial infarction on mortality from all causes and from coronary heart disease in men. J Am Coll Cardiol. 2002;40(5):954-60.

7. Hu FB, Stampfer MJ, Solomon CG, Liu S, Willett WC, Speizer FE, et al. The impact of diabetes mellitus on mortality from all causes and coronary heart disease in women: 20 years of follow-up. Arch Intern Med. 2001;161(14):1717-23.

8. Takeshita S, Zheng LP, Brogi E, Kearney M, Pu LQ, Bunting S, et al. Therapeutic angiogenesis. A single intraarterial bolus of vascular endothelial growth factor augments revascularization in a rabbit ischemic hind limb model. J Clin Invest. 1994;93(2):662-70.

9. Kawamoto A. Intramyocardial transplantation of autologous endothelial progenitor cells for therapeutic neovascularization of myocardial ischemia. Circulation. 2002;107(3):461-8

10. Tateishi-Yuyama E, Matsubara H, Murohara T, Ikeda U, Shintani S, Masaki H, et al. Therapeutic angiogenesis for patients with limb ischaemia by autologous transplantation of bone-marrow cells: a pilot study and a randomised controlled trial. Lancet. 2002;360(9331):427-35.

11. Ferrara N, Gerber HP, LeCouter J. The biology of VEGF and its receptors. Nat Med. 2003:9(6):669-76.

12. Giacca M, Zacchigna S. VEGF gene therapy: therapeutic angiogenesis in the clinic and beyond. Gene Ther. 2012;19(6):622-9.

13. Eichmann A, Simons M. VEGF signaling inside vascular endothelial cells and beyond. Curr Opin Cell Biol. 2012;24(2):188-93.

14. Wood JM, Bold G, Buchdunger E, Cozens R, Ferrari S, Frei J, et al. PTK787/ZK 222584, a novel and potent inhibitor of vascular endothelial growth factor receptor tyrosine kinases, impairs vascular endothelial growth factor-induced responses and tumor growth after oral administration. Cancer Res. 2000;60(8):2178-89.

15. Simons M. Angiogenesis: where do we stand now? Circulation. 2005;111(12):1556-66.

16. Jolicoeur EM, Granger CB, Fakunding JL, Mockrin SC, Grant SM, Ellis SG, et al. Bringing cardiovascular cell-based therapy to clinical application: perspectives based on a National Heart, Lung, and Blood Institute Cell Therapy Working Group meeting. Am Heart J. 2007;153(5):732-42.

17. Moriya J, Minamino T, Tateno K, Shimizu N, Kuwabara Y, Sato Y, et al. Longterm outcome of therapeutic neovascularization using peripheral blood mononuclear cells for limb ischemia. Circ Cardiovasc Interv. 2009:2(3):245-54.

18. Moriya J, Ferrara N. Inhibiting the response to VEGF in diabetes. Sci Signal. 2014;7(307):pe1-pe1.

19. Warren CM, Ziyad S, Briot A, Der A, Iruela-Arispe ML. A ligand-independent VEGFR2 signaling pathway limits angiogenic responses in diabetes. Sci Signal. 2014;7(307):ra1-ra1.

20. Fredriksson L, Li H, Eriksson U. The PDGF family: four gene products form five dimeric isoforms. Cytokine Growth Factor Rev. 2004;15(4):197-204.

21. Li X, Ponten A, Aase K, Karlsson L, Abramsson A, Uutela M, et al. PDGF-C is a new protease-activated ligand for the PDGF alpha-receptor. Nat Cell Biol. 2000:2(5):302-9.

22. LaRochelle WJ, Jeffers M, McDonald WF, Chillakuru RA, Giese NA, Lokker NA, et al. PDGF-D, a new protease-activated growth factor. Nat Cell Biol. 2001;3(5):517-21.

23. Dimmeler S. Platelet-derived growth factor CC-a clinically useful angiogenic factor at last? N Engl J Med. 2005;352(17):1815-6.
24. Crawford Y, Kasman I, Yu L, Zhong C, Wu X, Modrusan Z, et al. PDGF-C mediates the angiogenic and tumorigenic properties of fibroblasts associated with tumors refractory to anti-VEGF treatment. Cancer Cell. 2009;15(1):21-34.

25. Hou X, Kumar A, Lee C, Wang B, Arjunan P, Dong L, et al. PDGF-CC blockade inhibits pathological angiogenesis by acting on multiple cellular and molecular targets. Proc Natl Acad Sci U S A. 2010;107(27):12216-21.

26. Li XR, Tjwa M, Moons L, Fons P, Noel A, Ny A, et al. Revascularization of ischemic tissues by PDGF-CC via effects on endothelial cells and their progenitors. J Clin Investig. 2005;115(1):118-27.

27. Moriya J, Wu X, Zavala-Solorio J, Ross J, Liang XH, Ferrara N. Platelet-derived growth factor $\mathrm{C}$ promotes revascularization in ischemic limbs of diabetic mice. J Vasc Surg. 2014;59(5):1402-9. e1404.

28. Varma S, Lal BK, Zheng R, Breslin JW, Saito S, Pappas PJ, et al. Hyperglycemia alters PI3K and Akt signaling and leads to endothelial cell proliferative dysfunction. Am J Physiol Heart Circ Physiol. 2005;289(4):H1744-51.

29. Mochly-Rosen D, Das K, Grimes KV. Protein kinase C, an elusive therapeutic target? Nat Rev Drug Discov. 2012;11(12):937-57.

30. Geraldes $P$, King GL. Activation of protein kinase $C$ isoforms and its impact on diabetic complications. Circ Res. 2010;106(8):1319-31.

31. Moriya J, Minamino T, Tateno K, Okada S, Uemura A, Shimizu I, et al. Inhibition of semaphorin as a novel strategy for therapeutic angiogenesis. Circ Res. 2010;106(2):391-8.

32. Yokoyama M, Okada S, Nakagomi A, Moriya J, Shimizu I, Nojima A, et al. Inhibition of endothelial p53 improves metabolic abnormalities related to dietary obesity. Cell Rep. 2014;7(5):1691-703.

33. Toullec D, Pianetti $P$, Coste $H$, Bellevergue $P$, Grand-Perret $T$, Ajakane M, et al. The bisindolylmaleimide GF $109203 X$ is a potent and selective inhibitor of protein kinase C. J Biol Chem. 1991;266(24):15771-81.

34. Chang JT, Lu YC, Chen YJ, Tseng CP, Chen YL, Fang CW, et al. hTERT phosphorylation by PKC is essential for telomerase holoprotein integrity and enzyme activity in head neck cancer cells. Br J Cancer. 2006;94(6):870-8.

35. Chen H, Gu X, Liu Y, Wang J, Wirt SE, Bottino R, et al. PDGF signalling controls age-dependent proliferation in pancreatic beta-cells. Nature. 2011;478(7369):349-55.

36. Gui C, Li SK, Nong QL, Du F, Zhu LG, Zeng ZY. Changes of serum angiogenic factors concentrations in patients with diabetes and unstable angina pectoris. Cardiovasc Diabetol. 2013;12:34.

37. Lizotte F, Pare M, Denhez B, Leitges M, Guay A, Geraldes P. PKCdelta impaired vessel formation and angiogenic factor expression in diabetic ischemic limbs. Diabetes. 2013;62(8):2948-57.

38. Haidari M, Zhang W, Willerson JT, Dixon RA. Disruption of endothelial adherens junctions by high glucose is mediated by protein kinase C-beta-dependent vascular endothelial cadherin tyrosine phosphorylation. Cardiovasc Diabetol. 2014;13:112.

39. Harrington EO, Loffler J, Nelson PR, Kent KC, Simons M, Ware JA. Enhancement of migration by protein kinase $C$ and inhibition of proliferation and cell cycle progression by protein kinase $C$ in capillary endothelial cells. J Biol Chem. 1997;272(11):7390-7.

40. Xu H, Czerwinski P, Hortmann M, Sohn HY, Forstermann U, Li H. Protein kinase $C$ alpha promotes angiogenic activity of human endothelial cells via induction of vascular endothelial growth factor. Cardiovasc Res. 2008;78(2):349-55.

41. Corti F, Finetti F, Ziche M, Simons M. The syndecan-4/protein kinase Calpha pathway mediates prostaglandin E2-induced extracellular regulated kinase (ERK) activation in endothelial cells and angiogenesis in vivo. J Biol Chem. 2013;288(18):12712-21

42. Rask-Madsen C, King GL. Differential regulation of VEGF signaling by PKC-alpha and PKC-epsilon in endothelial cells. Arterioscler Thromb Vasc Biol. 2008;28(5):919-24.

43. Moncada de la Rosa C, Radziwon-Balicka A, El-Sikhry H, Seubert J, Ruvolo PP, Radomski MW, et al. Pharmacologic protein kinase Calpha inhibition uncouples human platelet-stimulated angiogenesis from collagen-induced aggregation. J Pharmacol Exp Ther. 2013;345(1):15-24. 